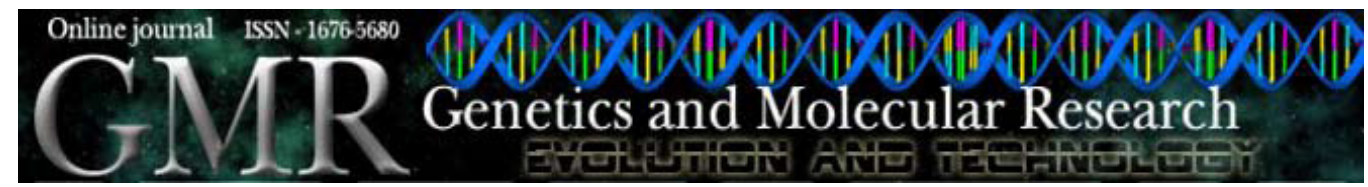

Case Report

\title{
A novel COMP mutation in an Inuit patient with pseudoachondroplasia and severe short stature
}

\author{
A.M. Elliott ${ }^{1,2,3}$, P. Bocangel ${ }^{1}$, M.H. Reed ${ }^{2,3,4}$ and C.R. Greenberg ${ }^{1,2,3}$ \\ ${ }^{1}$ WRHA Program of Genetics and Metabolism, University of Manitoba, \\ Winnipeg, Manitoba, Canada \\ ${ }^{2}$ Department of Paediatrics and Child Health, University of Manitoba, \\ Winnipeg, Manitoba, Canada \\ ${ }^{3}$ Department of Biochemistry and Medical Genetics, University of Manitoba, \\ Winnipeg, Manitoba, Canada \\ ${ }^{4}$ Department of Diagnostic Imaging, University of Manitoba, Winnipeg, \\ Manitoba, Canada \\ Corresponding author: A.M. Elliott \\ E-mail: aelliott@hsc.mb.ca
}

Genet. Mol. Res. 9 (3): 1785-1790 (2010)

Received May 28, 2010

Accepted July 18, 2010

Published September 8, 2010

DOI 10.4238/vol9-3gmr897

\begin{abstract}
Pseudoachondroplasia (PSACH) is an autosomal dominant skeletal dysplasia, generally identified clinically at two years of age due to decreased linear growth and a waddling gait. Radiographic features include small and irregular epiphyses, with metaphyseal changes of the long bones and characteristic vertebral changes. Mutations in the COMP gene cause PSACH and some cases of multiple epiphyseal dysplasia. Mutations generally cluster in the calmodulin-like repeat regions of the gene. Mutations in exon 13 (encoding the seventh calmodulin-like repeat) have been associated with severe short stature (-6 SD) in PSACH. We examined an Inuit boy with PSACH and severe short stature. Height essentially remained at $-1 \mathrm{SD}$ on the PSACH growth curve (-7.5 SD on a normal growth curve at 10.5 years). Analysis of $C O M P$ in our patient revealed a previously undescribed heterozygous $\mathrm{A}>\mathrm{T}$ substitution in exon 8 , at nucleotide
\end{abstract}


812. This change in the sequence resulted in replacement of a highly conserved and negatively charged aspartic acid with an uncharged, hydrophobic valine at amino acid position 271. Both unaffected parents were negative for this genetic change. This exon encodes the first calmodulin-like repeat, which has not been previously implicated in severe short stature. We propose that this novel missense substitution is responsible for the phenotype of this patient.

Key words: Pseudoachondroplasia; COMP; Skeletal dysplasia; PSACH

\section{INTRODUCTION}

Pseudoachondroplasia (PSACH; MIM\#177170) is an autosomal dominant skeletal dysplasia characterized by disproportionate short stature due to short limbs. Affected patients have normal birth length and are usually diagnosed at 2 years of age, when there is a deceleration in linear growth and a waddling gait is identified. Intellect is normal and there is no craniofacial involvement. There is ligamentous laxity and limb deformity is not uncommon. Early osteoarthritis is also a feature. Radiographic findings include abnormalities of the tubular bones, with metaphyseal and epiphyseal involvement and anterior beaking of the vertebral bodies (Unger and Hecht, 2001).

PSACH is a relatively genetically homogeneous disorder due to mutations in the cartilage oligomeric matrix protein (COMP) gene (Briggs et al., 1995; Hecht et al., 1995). Multiple epiphyseal dysplasia (MED; MIM\#132400), a genetically heterogeneous disorder, also results from COMP mutations in some patients. The COMP protein consists of an $\mathrm{N}$-terminal domain that is involved in pentamer assembly, four epidermal growth factor-like repeats, eight type III calmodulin-like repeats (CLRs) and a C-terminal globular domain. Most mutations in the COMP gene have been localized to the CLR regions, while others occur in the C-terminal globular domain (Oldberg et al., 1992; Newton et al., 1994; Efimov et al., 1994; Mabuchi et al., 2003; Kennedy et al., 2005). Severe short stature (-6 standard deviation, SD) in PSACH has been associated with mutations in exon 13 (encoding CLR7) (Mabuchi et al., 2003). Here, we report the clinical, radiographic and molecular features of an Inuit male patient with PASCH and severe short stature (-7.5 SD) who has a previously undescribed COMP mutation in exon 8 , which encodes CLR1. This mutation was absent in his unaffected parents.

\section{CLINICAL REPORT}

At the time of this report, the patient is $119 / 12$ years of age. He was born to a nonconsanguineous 29-year-old G7P6SA1 Inuit female and a 30-year-old Inuit male. The pregnancy was complicated by myasthenia gravis in the mother, which was not treated. Review of the family history was negative for short stature or other skeletal abnormalities. Birth parameters were not available. At 7 days of age, the patient's height was $49 \mathrm{~cm}$ (50th percentile), weight $3.15 \mathrm{~kg}$ (25th percentile) and occipitofrontal circumference (OFC) $34.2 \mathrm{~cm}$ (25th percentile). At 6 months of age, height was $62.8 \mathrm{~cm}$ (5th percentile), weight $7 \mathrm{~kg}$ (25th percentile) and OFC $45.5 \mathrm{~cm}$ (90th percentile). By 12 months of age, height was $67 \mathrm{~cm}$ ( $<5$ th percentile; -3.3 
SD), weight $8.46 \mathrm{~cm}$ (5th percentile) and $\mathrm{OFC} 47.5 \mathrm{~cm}$ ( $>50$ th percentile).

The patient was $29 / 12$ years of age when first referred for genetics evaluation regarding the possibility of a skeletal dysplasia. He was $77 \mathrm{~cm}$ ( $<5$ th percentile; $-4.7 \mathrm{SD}), 10.3 \mathrm{~kg}$ ( $<5$ th percentile; $-2.9 \mathrm{SD}$ ) and $48.2 \mathrm{~cm}$ (20th percentile) for height, weight and OFC, respectively. Physical examination revealed obvious disproportionate short stature with short limbs. No facial dysmorphism was noted. The ears were normal in shape. A narrow chest was noted. Examination of his hands showed brachydactyly and bilateral clinodactyly of the fifth digit. No trident positioning of the fingers was noted. The thumbs were not deviated. There were no abnormalities noted in the cardiovascular, abdominal and genitourinary systems. Marked genu varum as well as a waddling gait were noted. Investigations included a complete blood count, serum immunoglobulins, urine for mucopolysaccharides, and a genetic skeletal survey. All blood and urine investigations were normal and ruled out MPS IV and cartilage hair hypoplasia syndrome. Review of radiographs revealed features suggestive of PSACH including: anterior beaking of the vertebral bodies, small and irregular epiphyses, irregular metaphyses, and brachydactyly (Figure 1A-C).
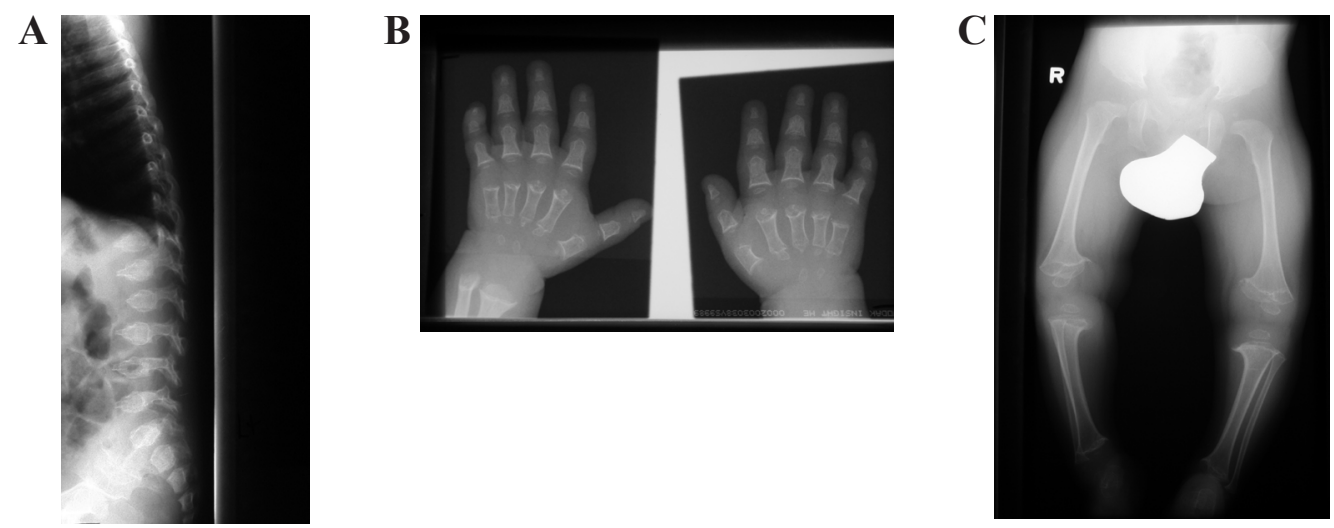

Figure 1. Radiographs at 33 months of age. A. Lateral spine. Anterior beaking (tonguing) of the vertebral bodies, characteristic of pseudoachondroplasia, is present. There is platyspondyly and slight variation in the size of the vertebral bodies. B. Bilateral PA of hands. There is generalized brachydactyly. There is widening of the metacarpals with severe shortening. There is metaphyseal involvement (e.g., cupping of proximal phalanges, widening and sclerosis of metacarpals). Pseudoepiphyses are present bilaterally. The epiphyses and carpal bones are small and irregular (carpal bone age is between 3 and 6 months). C. AP of pelvis and lower extremities. The capital femoral epiphyses are barely visible. There is shortening of the long bones. The metaphyses of the distal femurs, proximal tibias and fibulae are particularly widened, flared and sclerotic. The epiphyses are small and irregular. Fibular overgrowth (distally) is present. There is severe genu valgum.

At the age of 4 years, he started wearing leg braces. At 6 years of age, bilateral tibial and fibular osteotomies were performed. At 9 years of age, his height was $92 \mathrm{~cm}(-7.5 \mathrm{SD} ;-1$ SD on PSACH curve) and his weight $19.2 \mathrm{~kg}(-3.2 \mathrm{SD})$ (Horton et al., 1982). Bilateral asymmetry of the femurs and a windswept appearance to his lower extremities were noted. His hand joints were loose. Redundant skin was noted on his hands (Figure 2A,B). At 10 6/12 years of age, height was $94 \mathrm{~cm}(-7.6 \mathrm{SD} ;-1 \mathrm{SD}$ on PSACH curve), weight $21 \mathrm{~kg}(-3.4 \mathrm{SD})$ and OFC was 54.6 ( $\sim 90$ th centile). His upper to lower segment ratio was increased (1.7) and arm span was $79 \mathrm{~cm}$. Hand measurements revealed severe uniform brachydactyly (with a total length of 
$9.6 \mathrm{~cm}$, middle digit $4 \mathrm{~cm}$; both $3 \mathrm{rd}$ centile for a $26 / 12$ years old). Cognitive development was normal. Subsequent radiographs taken at $116 / 12$ years show an abnormal pelvis and progression of disease (Figure 3A,B). He lives in a remote Inuit community with a population of 748 (Statistics Canada 2006 census) in Nunavut, Canada. The limited population, geographic and social isolation make access to services for children with special needs a challenge but our patient to date is well accepted and integrated into his community. He is transported by air to Children's Hospital Winnipeg for acute medical care and subspecialty management.
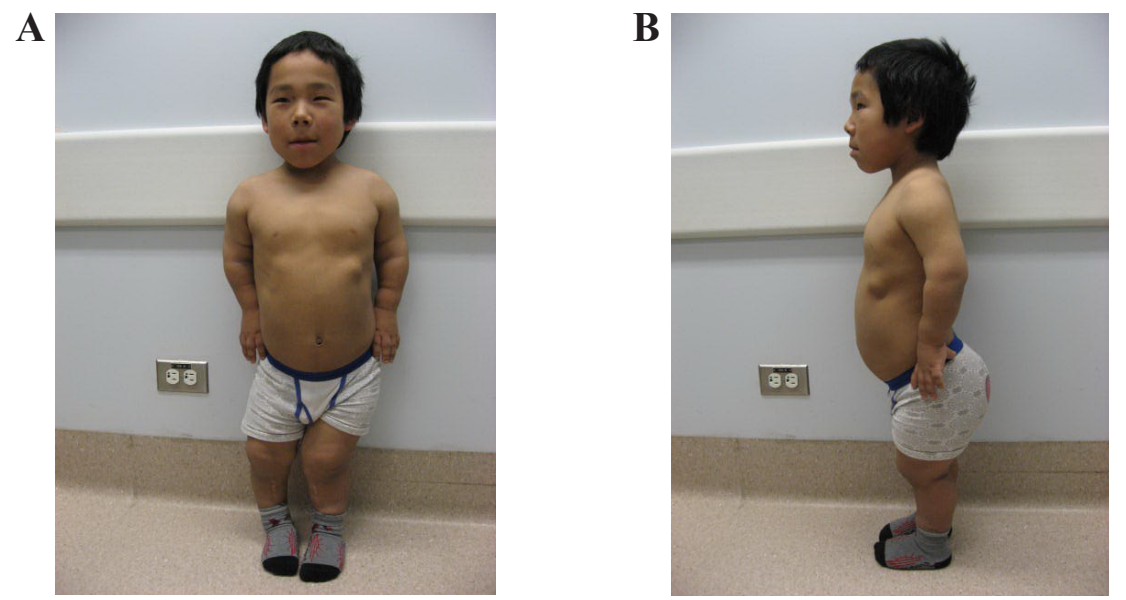

Figure 2. Full body photograph [AP (A) and lateral (B)] at 9 years of age showing disproportionate short limbed dwarfism and a windswept appearance of the lower extremities. Scars from surgical procedures are evident. There is no craniofacial involvement.
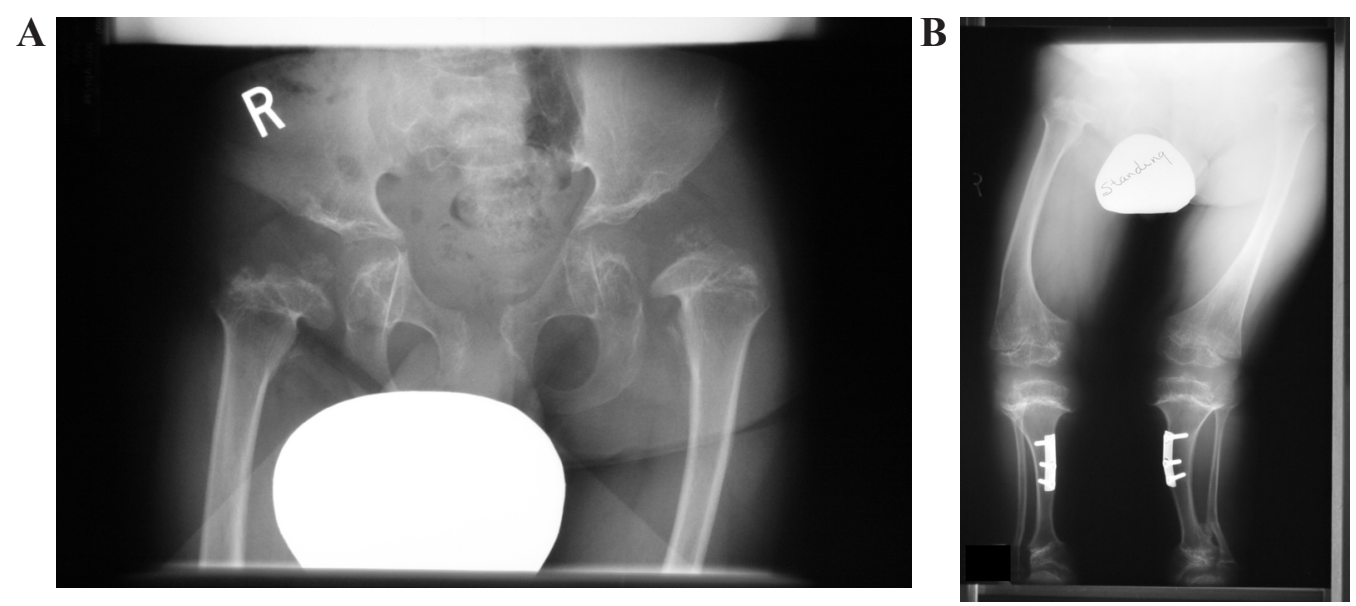

Figure 3. Follow-up radiographs at $116 / 12$ years. A. Pelvis. There is severe delay and irregularity in ossification of the proximal femoral epiphyses. The acetabulae are poorly formed and there is splaying of the ilia. The iliac wings are small and flared. The triradiate cartilage is abnormally wide and the femoral necks are short. There are small irregular centers of ossification for the lesser trochanters. B. Lower extremities. The bones remain short. The metaphyses are still flared and irregular. There is still distal fibular overgrowth. The genu valgum has been corrected surgically. There is distal ankylosis of the left tibia and fibula as a result of the surgery. 


\section{MOLECULAR STUDIES}

Initially, genomic DNA from the patient's specimen was polymerase chain reaction (PCR)-amplified for analysis of exons 14-18 of the COMP gene and no mutations were found. Subsequent analysis of exons 1-8, 15 and 17-19 of the COMP gene and their flanking splice sites was performed. Bi-directional sequence was obtained and DNA sequence was analyzed and compared to the published gene sequence. Genetic analysis revealed a novel heterozygous $\mathrm{A}>\mathrm{T}$ nucleotide substitution in exon 8 , resulting in the replacement of an aspartic acid codon (GAC) with a valine codon (GTC) at amino acid position 271: mutation denoted c.812A $>$ T at the cDNA level or p.ASP271Val (D271V) at the protein level. The result was confirmed by repeat sequence analysis. Both unaffected parents were negative for this genetic change.

\section{DISCUSSION}

The COMP gene consists of 19 exons and the majority of mutations are located within exons 8-14, which encode the type III CLRs (Briggs and Chapman, 2002). Most mutations are point mutations that result in amino acid substitutions or small in-frame deletions or insertions. The mechanism of pathogenesis is likely dominant negative in both PSACH and MED, as no mutations have been elucidated that would be expected to result in premature stop codons (Briggs and Chapman, 2002). The most common mutation is the D473del mutation in exon 13 encoding CLR7 (Deere et al., 1998; Ikegawa et al., 1998). Genotype/phenotype studies have revealed that mutations in CLR7 are associated with more severe short stature than those found in other CLRs. In a previous study, apart from one patient with a 553-bp deletion encompassing portions of exon 9 and intron 9, all patients with severe short stature (-6 SD) had mutations in CLR7 (Mabuchi et al., 2003). However, other patients with mutations CLR7 (exon 13) have not had extreme short stature (Nakashima et al., 2005).

The de novo sequence change found in our patient results in the replacement of a highly conserved and negatively charged aspartic acid with an uncharged, hydrophobic valine, and to our knowledge, has not been previously reported. Deere et al. (1999) reported nine novel mutations in COMP in patients with PSACH and MED. One of the PSACH patients (P1800) had a G863C substitution, which resulted in aspartate 271 being changed to a histidine. This mutation involves the same codon as our patient. Phenotypic information on their patient was not provided.

COMP is the fifth member of the thrombospondin (TSP) gene family of calcium binding proteins (Oldberg et al., 1992; Newton et al., 1994; Adams et al., 1995). All TSPs have highly conserved sequences (the CLRs) that represent a contiguous series of conformationally sensitive calcium-binding sites, thus, mutations in these repeats interfere with the protein's ability to bind calcium. The majority of COMP mutations ( $>85 \%$ ) occur in these domains reinforcing the importance of the CLRs to the integral structure of the protein (Unger and Hecht, 2001; Briggs and Chapman, 2002; Kennedy et al., 2005). Mutations in these domains likely interfere with protein folding and underlie the retention of mutated COMP in the rough endoplasmic reticulum of PSACH patient cells (Unger and Hecht, 2001; Kleerekoper et al., 2002; Kennedy et al., 2005). Recently, Posey et al. (2009) generated a mouse model that recapitulated human PSACH growth plate morphology, including retention of extracellular matrix proteins, intracellular matrix formation in the rER cisternae and increased chondrocyte apoptosis.

The novel mutation and phenotype of our patient illustrates that severe short stature is 
not limited to mutations in CLR7 and further reinforces the importance of the CLRs and their role in protein function.

\section{ACKNOWLEDGMENTS}

The authors thank the family for their participation. This research was supported in part by a grant from the Manitoba Institute of Child Health, Inc. We acknowledge the care and support provided by Dr. Micheal Moffatt and the Inuit Health Service of the J.A. Hildes Northern Medical Unit.

\section{REFERENCES}

Adams ME, Matyas JR, Huang D and Dourado GS (1995). Expression of proteoglycans and collagen in the hypertrophic phase of experimental osteoarthritis. J. Rheumatol. (Suppl) 43: 94-97.

Briggs MD and Chapman KL (2002). Pseudoachondroplasia and multiple epiphyseal dysplasia: mutation review, molecular interactions, and genotype to phenotype correlations. Hum. Mutat. 19: 465-478.

Briggs MD, Hoffman SM, King LM, Olsen AS, et al. (1995). Pseudoachondroplasia and multiple epiphyseal dysplasia due to mutations in the cartilage oligomeric matrix protein gene. Nat. Genet. 10: 330-336.

Deere M, Sanford T, Ferguson HL, Daniels K, et al. (1998). Identification of twelve mutations in cartilage oligomeric matrix protein (COMP) in patients with pseudoachondroplasia. Am. J. Med. Genet. 80: 510-513.

Deere M, Sanford T, Francomano CA, Daniels K, et al. (1999). Identification of nine novel mutations in cartilage oligomeric matrix protein in patients with pseudoachondroplasia and multiple epiphyseal dysplasia. Am. J. Med. Genet. 85: 486-490.

Efimov VP, Lustig A and Engel J (1994). The thrombospondin-like chains of cartilage oligomeric matrix protein are assembled by a five-stranded alpha-helical bundle between residues 20 and 83. FEBS Lett. 341: 54-58.

Hecht JT, Nelson LD, Crowder E, Wang Y, et al. (1995). Mutations in exon 17B of cartilage oligomeric matrix protein (COMP) cause pseudoachondroplasia. Nat. Genet. 10: 325-329.

Horton WA, Hall JG, Scott CI, Pyeritz RE, et al. (1982). Growth curves for height for diastrophic dysplasia, spondyloepiphyseal dysplasia congenita, and pseudoachondroplasia. Am. J. Dis. Child 136: 316-319.

Ikegawa S, Ohashi H, Nishimura G, Kim KC, et al. (1998). Novel and recurrent COMP (cartilage oligomeric matrix protein) mutations in pseudoachondroplasia and multiple epiphyseal dysplasia. Hum. Genet. 103: 633-638.

Kennedy J, Jackson GC, Barker FS, Nundlall S, et al. (2005). Novel and recurrent mutations in the C-terminal domain of COMP cluster in two distinct regions and result in a spectrum of phenotypes within the pseudoachondroplasia multiple epiphyseal dysplasia disease group. Hum. Mutat. 25: 593-594.

Kleerekoper Q, Hecht JT and Putkey JA (2002). Disease-causing mutations in cartilage oligomeric matrix protein cause an unstructured $\mathrm{Ca}^{2+}$ binding domain. J. Biol. Chem. 277: 10581-10589.

Mabuchi A, Manabe N, Haga N, Kitoh H, et al. (2003). Novel types of COMP mutations and genotype-phenotype association in pseudoachondroplasia and multiple epiphyseal dysplasia. Hum. Genet. 112: 84-90.

Nakashima E, Mabuchi A, Kubota M, Ishikiriyama S, et al. (2005). Novel and recurrent exon 13 mutations of COMP in pseudoachondroplasia. Am. J. Med. Genet. A 132A: 108-109.

Newton G, Weremowicz S, Morton CC, Copeland NG, et al. (1994). Characterization of human and mouse cartilage oligomeric matrix protein. Genomics 24: 435-439.

Oldberg A, Antonsson P, Lindblom K and Heinegard D (1992). COMP (cartilage oligomeric matrix protein) is structurally related to the thrombospondins. J. Biol. Chem. 267: 22346-22350.

Posey KL, Veerisetty AC, Liu P, Wang HR, et al. (2009). An inducible cartilage oligomeric matrix protein mouse model recapitulates human pseudoachondroplasia phenotype. Am. J. Pathol. 175: 1555-1563.

Unger S and Hecht JT (2001). Pseudoachondroplasia and multiple epiphyseal dysplasia: New etiologic developments. Am. J. Med. Genet. 106: 244-250. 